\title{
Degradation Measurement of Kinect Sensor Under Fast Neutron Beamline
}

\author{
Zeba Khanam, Sangeet Saha, Bilal Aslam, Xiaojun Zhai, Shoaib Ehsan, Carlo Cazzaniga, Christopher \\ Frost, Rustam Stolkin and Klaus McDonald-Maier
}

\begin{abstract}
The neutron-induced degradation of depth images acquired using a Kinect sensor is investigated through the standard metrics. The evaluated metrics indicate saturation of degradation after several hours of exposure, which is recovered through annealing.
\end{abstract}

\section{INTRODUCTION}

\section{A. Motivation}

The present decade has witnessed an increment in number of sites hosting extreme environments, which require immediate inspection and exploration. The conditions prevalent in such sites vary significantly and may be characterized by extreme temperature and radiation levels. Thus, direct human exploration in these hostile conditions can be fatal to human life. Apart from resulting in large number of causalities, this daunting task is interlaced with other challenges, which are time intensive and complex environmental catastrophes.

In this paper, we have narrowed down the scope by focusing on sites with extreme environments present in nuclear facilities. The precarious characteristics of these facilities are presence of radioactive elements and often also extreme temperature. In order to overcome the challenges, robots have been deployed for exploration and inspections [1]. The depth sensor was used by the robot as an eye to perceive and construct a 2D indoor map of the nuclear facility. However, the radioactive elements in these hazardous sites had initiated degradation of depth sensors causing a hurdle in exploration. Therefore, there is a dire need to further model the degradation induced in depth sensors.

\section{B. Existing Work}

There have been various attempts in recent past to model the degradation of sensors due to radiation exposure. The exposed radiation source can be broadly categorized into two categories mainly: 1) ray based and 2) particle based. Extensive researches have highlighted that radiation exposure impinge semiconductor devices [2]-[3]. The focal point of radiation

Zeba Khanam, Sangeet Saha, Bilal Aslam, Xiaojun Zhai, Shoaib Ehsan and Klaus McDonald-Maier are with Embedded and Intelligent Systems Lab, University of Essex, Colchester CO4 3SQ, U.K (zeba.khanam@essex.ac.uk, sangeet.saha@essex.ac.uk, ba18997@essex.ac.uk, xzhai@essex.ac.uk, sehsan@essex.ac.uk, kdm@essex.ac.uk)

Carlo Cazzaniga and Christopher Frost is with ISIS Facility, Rutherford Appleton Laboratory, Chilton, Didcot, UK (carlo.cazzaniga@stfc.ac.uk, christopher.frost@stfc.ac.uk)

Rustam Stolkin is with Extreme Robotics Lab (ERL), University of Birmingham, Birmingham B15 2TT, U.K (r.stolkin@cs.bham.ac.uk)

Manuscript received xxxx; revised $\mathrm{xxxx}$ induced degradation measurement has been on custom camera sensors. However, there are several of works focusing on depth sensors. Most of the studies have reported failure of depth sensors due to the exposure of gamma rays [4]-[5]. A recent study also investigates the impact of gamma rays, while the depth sensor is operational [6]. There are significant changes observed in the sensors' static sensitivity depending on dose.

The aforementioned experimental studies target postradiation-disaster environments such as the FukushimaDaiichii nuclear power plant. These sites can consist of a mixture of radioactive elements, like alpha, beta, gamma and neutron [7]-[8]. Gamma rays and neutrons are of a major concern, due to their ability to impact semiconductor devices with high penetration range and long time duration persistence [9]. Since major works have focussed on experimental evaluation of degradation of commercial depth sensors due to gamma radiation exposure, we focus on a different and important direction i.e., measurement of degradation of depth sensors due to fast neutron exposure. To best of the knowledge of the authors, their is no prior concrete work on evaluation of degradation in depth sensors due to neutron exposure. However in a recent experimental studies [10], neutron based radiation testing of different commercially available cameras have been carried out. It was reported that the commercial cameras permanently failed at relatively low total integrated dose of neutron. Another interesting study [11] has investigated displacement damage and single event effects on CMOS image sensors induced by neutron irradiation.

\section{Contributions}

We measure the degradation induced by radiation in depth images, when the sensor is exposed to radioactive particles with high energy like fast neutrons. The aim of this work is to characterize the radiation induced degradation in depth images as a function of exposure time. The depth sensor chosen for irradiation is Microsoft Kinect which has been used previously by robots for radiation facility exploration [1].

Firstly, the degradation of the Kinect sensor is observed as noise in acquired depth images during radiation experiments. Then, induced degradation is classified as displacement damage and single event effect. We also analyse the quality of irradiated depth images using Image Quality Assessment metrics. The experimental evaluations have been illustrated in Figure 1. This work will serve as a building block in assisting robots to perceive depth in a nuclear sites in a better fashion. 


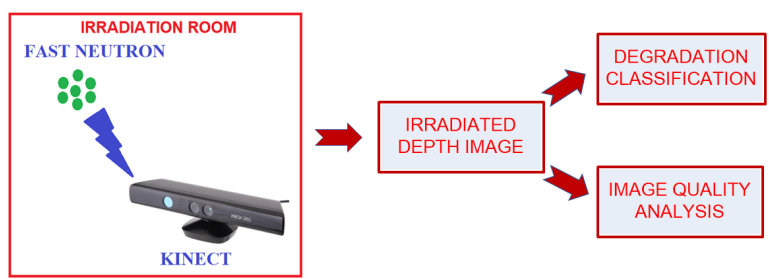

Fig. 1: Experimental evaluation

\section{EXPERIMENTAL SETUP}

The exposure of the Kinect sensor to fast neutron was carried out by conducting experiments with fast neutrons at ChipIr facility in ISIS, Rutherford Appleton Laboratory, Didcot, UK [12]. This facility provides a neutron spectrum which is suitable to emulate effects of terrestrial neutrons in any given electronic sensor. The ChipIr neutron flux (with $10 \mathrm{MeV}<E_{n}<800 \mathrm{MeV}$ ) has been measured to be $5 \times 10^{6} \mathrm{~cm}^{-2} \mathrm{~s}^{-1}$. The Kinect sensor was irradiated for about 20 hours at ChipIr, that amounts to 3.5 million years of natural exposure. Figure 2a depicts our experimental setup on the beam lines at ChipIr facility.

The Kinect sensor is capable of capturing RGB, Infra-Red (IR), depth, skeleton and audio streams simultaneously at a given time instance. The IR emitter projects a speckle pattern over the given scene. The reflected pattern is captured using IR camera. This allows the depth of objects in scene to be gauged by correlation [13]. Thus, neutron beam is focussed on IR emitter first for 10 hours. Then, an annealing process at room temperature for a time period of 10 hours is performed. This is followed by exposure of neutron beam focussed at IR camera for another 10 hours.

\section{RESULTS AND DISCUSSION}

The main objective of our research is to measure the degradation effect on the Kinect depth images due to neutron exposure. The degradation manifest as noises in depth images which is characterized as a function of radiation exposure time. In order to realize this ambition, we first estimate the noise and then classify it into two categories: 1) displacement damage (permanent degradation) and 2) single event effect (transient degradation). Further, we analyse the quality of irradiated depth image by full reference Image Quality Assessment (IQA) metrics that operates in spatial domain. The prime requirement of these metrics is to obtain a reference image.

\section{A. Reference Image}

The reference image, $I_{r e f}$, is the depth image of static scene captured by the Kinect without the radiation exposure. It is worth noting that there are many noises induced in Kinect depth images due to multiple factors [13]. We aim to remove the prominent noises in depth images of any static scene.

For a given static scene, the depth values are expected to be steady. However, it was observed that the depth value for object points are unstable in multiple frames varying in time. This behaviour is temporal in nature and illustrated as salt and pepper noise in depth images [13]. Thus, we have used median filtering to remove these noises.

Let $I_{N R}$ be the set of depth images of static scenes collected without radiation. The reference image $I_{r e f}$ is obtained using:

$$
I_{N R}=\left\{I_{N R}^{0}, I_{N R}^{1}, \ldots, I_{N R}^{\eta-1}\right\}
$$

where $\eta$ is total number of images acquired after time interval $t$. Any given pixel $(i, j)$ in $I_{r e f}$ is obtained:

$$
I_{\text {ref }}(i, j)=\operatorname{Median}\left(I_{N R}^{0}(i, j), \ldots, I_{N R}^{\eta-1}(i, j)\right)
$$

where $i \in\{1, \ldots, m\}, j \in\{1, \ldots, n\}$ are spatial indices and $m \times n$ is the dimension of reference image $I_{r e f}$.

\section{B. Radiation Induced Noise}

After obtaining the reference image, we irradiate the Kinect sensor with neutron beam. Let $I_{R}$ be a set of depth images captured after Kinect is exposed to the neutron radiation. Each depth map in $I_{R}$ captures same static scene used in $I_{N R}$. Then $I_{R}$ can be formulated as:

$$
I_{R}=\left\{I_{R}^{0}, I_{R}^{1}, \ldots, I_{R}^{k-1}\right\}
$$

where $k$ is total number of images acquired after time interval $t$. The radiation induced noise, $N$, is calculated for any irradiated depth image $I_{R}^{l}$ as follows:

$$
N(i, j)=\left\{\begin{array}{cc}
1 & \left|I_{R}^{l}(i, j)-I_{\text {ref }}(i, j)\right|>T h \\
0 & \text { otherwise }
\end{array}\right.
$$

$I_{r e f}$ is the reference image calculated using Equation 2 with $m \times n$ as the resolution of depth images. Th is the threshold sampling value selected empirically in the experiment.

1) Classification: Past studies have demonstrated that neutron irradiation of semiconductor materials can dislocate atoms from their normal lattice location. This allows creation of Frenkel pair and divacancy [14]. Due to the dis-alignment of atoms in crystalline structure there are two effects observed in depth images which can be classified as:

- Displacement Damage $(D D)$ are set of pixels which undergo permanent damage.

- Single Event Effect $(S E E)$ are set of pixels which undergo transient damage.

To classify both the effects, we have proposed an algorithm (refer Algorithm 3). The algorithm compares spatial occurrence of noise pixels in depth images over a span of time.

The evolution of DD and SEE in Kinect depth sensors over the exposure period is shown in Figure 4. The displacement damage rises from being negligible in the initial hours to account for more than half of damaged pixels after four hours of irradiation. This saturates until there is a shift in focus of radiation from IR emitter to IR camera. The annealing process of 10 hour allows the Kinect sensor to recover from this displacement damage. After the focus shifts to the IR camera, the progressive increment in displacement damage under neutrons exposure is observed. In contrast, there is an upsurge in Single Event Effect (SEE) observed at initial exposure of neutron irradiation which declines over span of 


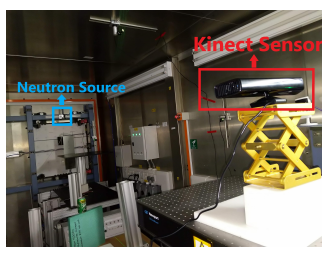

(a) Experimental Setup

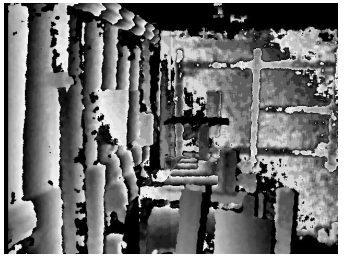

(b) Reference Image

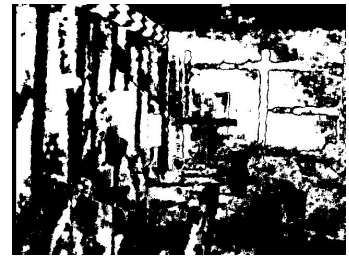

(c) Radiation Noise

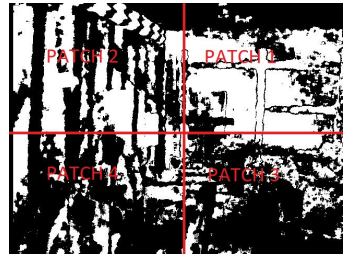

(d) Patch Division

Fig. 2: Experimental setup and observed noise

\section{Input:}

1. $I_{\text {ref }}$ : reference image.

2. $I_{R}$ : a set of irradiated images with time stamp.

Output: $D D, S E E$ : dispalcement damage and single event effect

\section{Begin:}

$L=N U L L$;

for each irradiated depth image $I_{R}^{l} \in I_{R}$ do

Calculate radiation noise $N$ for $I_{R}^{l}$ and $I_{\text {ref }}$ using

Equation 4;

Store the noise pixel positions in a list $L_{t}$ created at $\mathrm{t}$; $L=L \cup L_{t}$;

for each $L_{t} \in L$ do

Consider two consecutive lists $L_{t}$ and $L_{t+1}$; $\quad \triangleright$ where

$L_{t+1}$ denotes the list created from image with time stamp $t+1$

if a noise pixel appears in both the lists then

| Noise is classified as Displacement Damage; else

$L$ Noise is classified as Single Event Effect;

Fig. 3: Algorithm 1 (Displacement Damage and Single Event Effect)

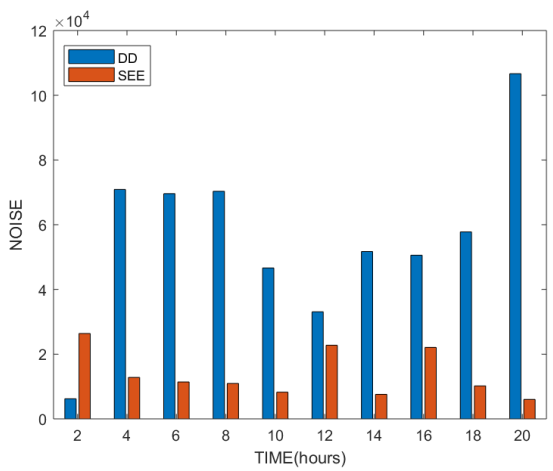

Fig. 4: Displacement Damage (DD) v/s Single Event Effect (SEE) observed over time

time. This allows us to conclude that when Kinect experiences an initial exposure to neutron there is a higher ratio of noise is SEE in comparison to $D D$. However, with passage of time, SEE decreases and $D D$ increases.

\section{IQA Metric}

The quality assessment has been carried out using following IQA metrics:.
1) Entropy: Entropy of an image is a statistical measure of randomness and is useful in characterization of texture. This can be defined as:

$$
H(I)=-\sum_{n=1}^{256} p_{n}(I) \times \log _{2}\left(p_{n}(I)\right)
$$

where $p_{n}(I)$ are probabilities associated with the bins of histogram of input image $I$. We use entropy to classify the texture of irradiated depth images. The random spatial occurrence of noise in depth images results in development of a coarse texture which leads to higher entropy. The entropy of depth maps are plotted as a function of radiation exposure time (refer Fig. 5). The plot illustrates that entropy increases with time, which is indicative of increment in noise. At initial exposure, entropy increases indicating start of degradation of depth image quality which saturates after four hour of accumulative exposure. However, the entropy of depth images, which are obtained after the annealing period decreases significantly, indicating recovery from displacement damage. This is in sync with the observation in Figure 4. With irradiation of neutron beam focused on IR emitter, a rise in entropy is observed which indicates further degradation of quality of depth map. To affirm these observation, we calculate other global IQA metrics used in literature [15].

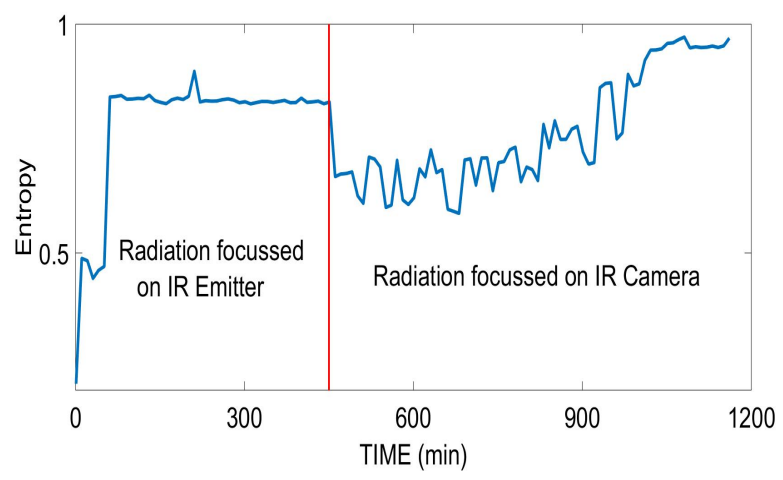

Fig. 5: Entropy as a function of radiation exposure time

2) Peak Signal to Noise Ratio (PSNR): PSNR is the ratio of the maximum possible power of a signal and noise. In our case, signal is the reference image and noise is the irradiated induced noise. This is formulated as:

$$
P S N R=20 \log _{10}\left(I_{\text {ref }}\right)-10 \log _{10}(N)
$$

Lower PSNR value is indicative of higher presence of radiation noise. 
3) Structural Similarity Index (SSIM): This metric in accordance to the human visual system assesses quality of a depth images by comparing three components: 1) luminance 2) contrast and 3) structure. Lower SSIM value is indicative of higher presence of radiation noise.

Figure 6 and Figure 7 depict PSNR and SSIM as a function of radiation exposure time, respectively. In contrast to entropy, PSNR and SSIM inversely decline as the noise increases. After analysing all the quality assessment metrics which incline towards similar inference, we can conclude two noteworthy observations. First, radiation induced noise saturates after four hours of continuous and focused radiation exposure. Second, the annealing process of 10 hours allow recovery from degradation noise.

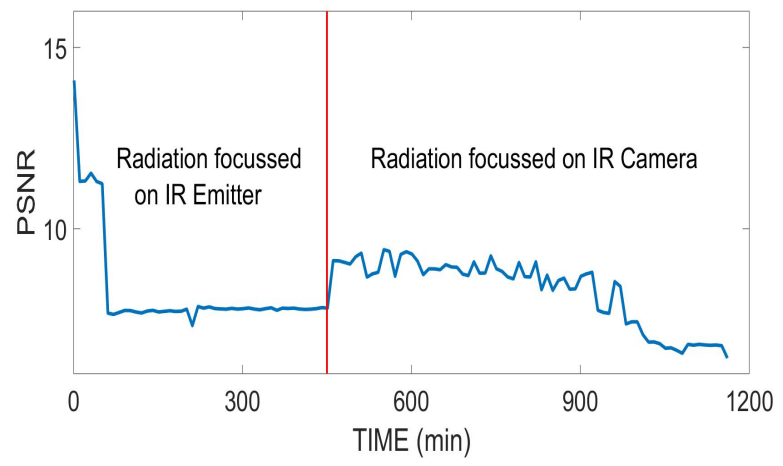

Fig. 6: PSNR Metric as a function of radiation exposure time

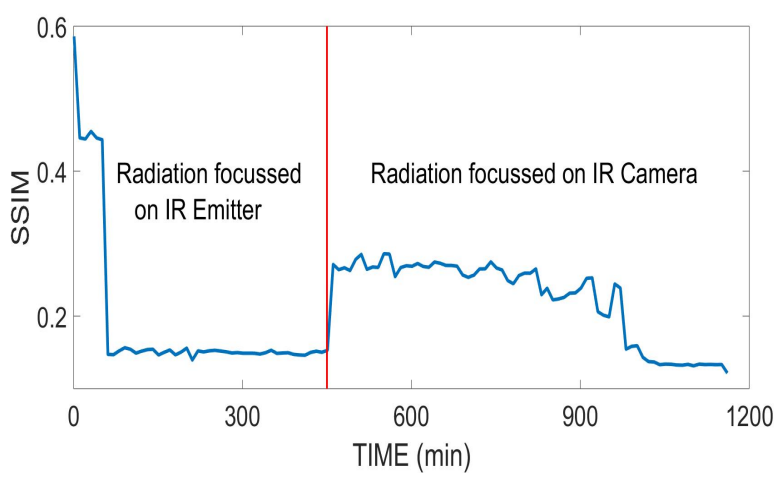

Fig. 7: SSIM Metric as a function of radiation exposure time

\section{CONCLUSION}

This paper takes a pertinent step practically by measuring the radiation induced degradation in Kinect sensors due to fast neutron exposure. The degradation is measured as noise in depth images and classified as single-event effect and displacement damage. The quality of irradiated depth images are also gauged using IQA metrics. This experimental study allows us to conclude that radiation induced degradation saturates after few hours of exposure and annealing process at room temperature triggers recovery of radiation induced degradation.

\section{Future DiRECTION}

This successful experimental study of characterization gives us a confidence to delve our research in following direction. The estimation of radiation induced noise in Figure $2 \mathrm{c}$ reveals a trend of noise occurrence as concentrated blobs. To investigate further, we plan to divide depth image into patches as illustrated in Figure 8. Any depth image can be divided into number of patches, ranging from two to its resolution size. Figure $2 \mathrm{~d}$ depicts division of depth images into four patches. An experimental analysis needs to be performed to determine the number of patches a depth image can be divided into, which will yield fruitful results.

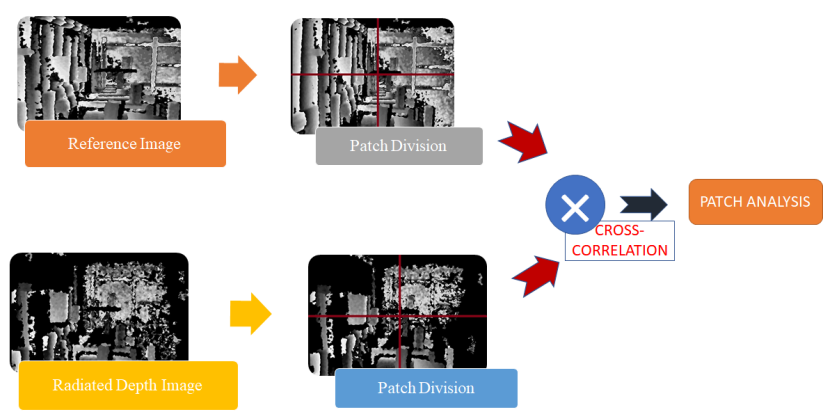

Fig. 8: Patch level analysis

The radiation induced noise can be characterized by calculating cross-correlation between the patch of irradiated image and the patch of reference image. This is indicative of similarity between radiation image patch and reference image patch.

The patch level evaluation will allow us to conclude if radiation noise occurs as a concentrated blobs in free space or objects. This will guide us to develop applications with recent computer vision techniques like Convolution Neural Networks in unaffected areas in depth images that can result in robot to perceive nuclear environment in more prolific manner.

\section{ACKNOWLEDGEMENT}

This work is supported by the UK Engineering and Physical Sciences Research Council through grants EP/R02572X/1 and EP/P017487/1.

\section{REFERENCES}

[1] B. Bird et al., "Radiological monitoring of nuclear facilities: Using the continuous autonomous radiation monitoring assistance robot," IEEE Robotics \& Automation Magazine, 2018.

[2] C. Virmontois and others., "Displacement damage effects due to neutron and proton irradiations on cmos image sensors manufactured in deep submicron technology," IEEE Transactions on Nuclear Science, vol. 57, no. 6, pp. 3101-3108, 2010.

[3] J. Howard et al., "Methodology for identifying radiation effects in robotic systems with mechanical and control performance variations," IEEE Transactions on Nuclear Science, vol. 66, no. 1, pp. 184-189, 2019.

[4] H. Igarashi et al., "Evaluation of sensors for mobile robots based on irradiation experiment," in 2012 IEEE/SICE International Symposium on System Integration (SII). IEEE, 2012, pp. 517-522.

[5] Z. Diggins et al., "Impact of gamma radiation on range finding sensor performance," in SENSORS, 2013 IEEE. IEEE, 2013, pp. 1-4.

[6] Z. J. Diggins et al., "Range-finding sensor degradation in gamma radiation environments," IEEE Sensors Journal, vol. 15, no. 3, pp. 1864 1871, 2015. 
[7] N. E. R. Headquarters, "Report of japanese government to the iaea ministerial conference on nuclear safety-the accident at tepco's fukushima nuclear power stations," http://www. kantei. go. jp/foreign/kan/topics/201106/iaea_houkokusho_e. html, 2011.

[8] F. Daiichi, "Ans committee report," A Report by The American Nuclear Society Special Committee on Fukushima, 2012.

[9] K. Nagatani et al., "Gamma-ray irradiation test of electric components of rescue mobile robot quince," in 2011 IEEE International Symposium on Safety, Security, and Rescue Robotics. IEEE, 2011, pp. 56-60.

[10] M. Dayton et al., "Radiation effects on active camera electronics in the target chamber at the national ignition facility," in Target Diagnostics Physics and Engineering for Inertial Confinement Fusion VI, vol. 10390. International Society for Optics and Photonics, 2017, p. 1039007.

[11] B. Aslam et al., "Degradation measurement of commercial camera sensors under fast neutron beamline," in Accepted for Proceedings of 28th Annual Single Event Effects (SEE) Symposium and Military and Aerospace Programmable Logic Devices (MAPLD) Workshop, 2019.

[12] C. Cazzaniga and C. D. Frost, "Progress of the scientific commissioning of a fast neutron beamline for chip irradiation," in Journal of Physics: Conference Series, vol. 1021, no. 1. IOP Publishing, 2018, p. 012037.

[13] T. Mallick et al., "Characterizations of noise in kinect depth images: A review," IEEE Sensors journal, vol. 14, no. 6, pp. 1731-1740, 2014.

[14] J. Srour and J. Palko, "Displacement damage effects in irradiated semiconductor devices," IEEE Transactions on Nuclear Science, vol. 60, no. 3, pp. 1740-1766, 2013.

[15] J. Fagerström, "Ionizing radiation effects on image sensors: Method on evaluation from an image quality perspective," 2016. 